
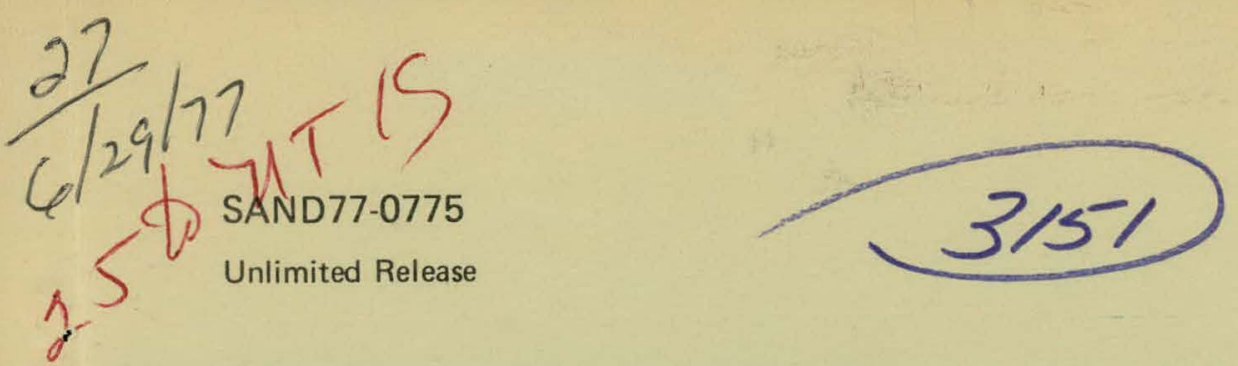

\title{
Evaluation of the Potential to Upgrade the Sandia Atomic lodine Laser SAIL-1 to Higher Output Energies
}

\author{
Merle E. Riley, Richard E. Palmer
}

Prepered by Sandia Laboratories, A lbuquarque, New Mexico 87115

and Livarmore, Califor nia 94550 for the United States Energy Research

and Development Administration under Contract AT(29-1)-789

Printed May 1977 


\section{DISCLAIMER}

This report was prepared as an account of work sponsored by an agency of the United States Government. Neither the United States Government nor any agency Thereof, nor any of their employees, makes any warranty, express or implied, or assumes any legal liability or responsibility for the accuracy, completeness, or usefulness of any information, apparatus, product, or process disclosed, or represents that its use would not infringe privately owned rights. Reference herein to any specific commercial product, process, or service by trade name, trademark, manufacturer, or otherwise does not necessarily constitute or imply its endorsement, recommendation, or favoring by the United States Government or any agency thereof. The views and opinions of authors expressed herein do not necessarily state or reflect those of the United States Government or any agency thereof. 


\section{DISCLAIMER}

Portions of this document may be illegible in electronic image products. Images are produced from the best available original document. 
Issued by Sandia Laboratories, operated for the United States Energy Research \& Development Administration by Sandia Corporation.

\section{NOTICE}

This report was prepared as an account of work sponsored by the United States Government. Neither the United States nor the United States Energy Research \& Development Administration, nor any of their employees, nor any of their contractors, subcontractors, or their employees, makes any warranty, express or implied, or assumes any legal liability or responsibility for the accuracy, completeness or usefulness of any information, apparatus, product or process disclosed, or represents that its use would not infringe privately owned rights.

SF 1004-DF (3-75) 
SAND-77-0775

Unlimited Release

Printed May 1977

EVALUATION OF THE POTENTIAL TO UPGRADE THE SANDIA ATOMIC IODINE IASER SAIH-1 TO HIGHER OUTPUT ENERGIES

M. E. Riley

Laser Theory Division 5211

R. E. Palmer

Advanced Laser-Physics Technology Division 5216

Sandia Laboratories

Albuquerque, New Mexico 87115

\begin{abstract}
The predicted output energy of the Sandia Atomic. Iodine Laser SAIL-1 is given for various numbers of preamplifier stages and for various small signal gains in each stage. Additional possibilities for further increasing the output energy are given.
\end{abstract}

Printed in the United States of America Available from

National Technical Information Service

U. S. Department of Commerce

5285 Port Royal Road

Springfield, VA 22161

Price: Printed Copy $\$ 4.00$; Microfiche $\$ 3.00$
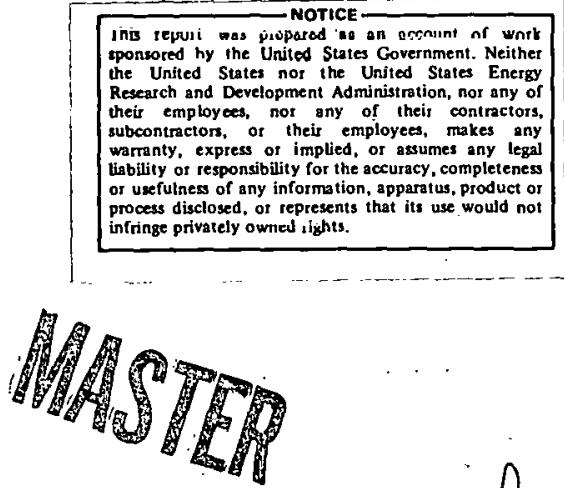
TABLE OF CONTENTS

Page

I. Introduction . . . . . . . . . . . . . . . . . 5

II. Theory ........................ . . . 6

A. Two-Level Radiation Transport Equations . . . . . 6

B. Atomic Iodine Transition Parameters . . . . . . . 8

C. Beam Propagation . . . . . . . . . . . . 9

III. Parametric Study of Energy Extraction in Upgraded SAIL-1 . 16

IV. Beam Quality Limitations . . . . . . . . . . . . . . 22

A. Prelase and Self-Oscillation........... 22

B. Target Feedback . . . . . . . . . . . . 23

C. Aperturing ................. 24

V. Additional Possibilities for Increasing the Output

Energy from SAIL-1 . . . . . . . . . . . . . . .

A. Two-Line Oscillator . . . . . . . . . . . . . .

B. Improved Pumping and Energy Storage in $75 \mathrm{~mm}$

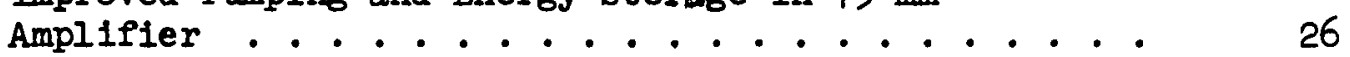

C. Cassegrainian Optics ............... . 27

Appendix: Correction to SLA-73-0976. . . . . . . . . 28 


\section{Introduction}

The atomic lodine laser is capable of producing output powers equal to I TW and output energies of several hundred joules. ${ }^{1,2}$ The atomic lodine laser at Sandia Laboratories, $\mathrm{SA} C-1$, is a moderate-sized system conslsting of an oscillator, a preamplifier, and a final main amplifier. In its present form, it has produced output energies of $10 \mathrm{~J}$ with output powers of 30-40 GW. If the output energy were somewhat higher, SAII-I could be an extremely useful research tool in the study of laser plasma interactions. The purpose of this report is to evaluate the expected energy output from SAIT-I for various numbers of additional preamplifier stages and for various gains in each stage.

In Section II the theoretical bases used to calculate the expected energy output are discussed. The results for many different system conflgurations are given in Section III. Section IV deals with a number of limiting conditions relating to beam quality and spurious outputs such as prelase and amplifier self-oscillation. Section $V$ mentions a few possibilities to increase the energy output even further, 1.e. a two-line oscillator, improved pumping, and the use of cassegrainian optics. 


\section{Theory}

\section{A. Two-Level Radiation Transport Equations}

An accurate description of the amplification of subnanosecond pulses in the atomic iodine system requires the Maxwell-Bloch theory, since the pulsewidth and the medium coherence decay time are comparable. ${ }^{3}$ However, if the pulsewidth is on the order of a nanosecond or longer, the Maxwe $\mathrm{J}_{\mathrm{n}}=$ Bloch equations can be approximated by the photon rate equations, Moreover, in most of the circumstances of interest here, the medium behaves like a two level atomic system with level degeneracy. The rate equations describing the evolution of the pulse and the medium are

$$
\begin{aligned}
& \left(\frac{\partial}{\partial z}+\frac{I}{c} \frac{\partial}{\partial t}\right) I=\frac{\partial I}{\partial z}=\sigma_{s e} I n \\
& \frac{\partial n}{\partial t}=\frac{\partial n}{\partial t}=-b \frac{I}{h \nu} \sigma_{s e^{n}} .
\end{aligned}
$$

$I=I(z, t)$ is the intensity in units of power per area; $z$ is axial distance;

$t$ is time; $t^{\prime}=t-z / c$ is the retarded time variable with $z^{\prime}=z$; $n$ is the inversion number density, defined in terms of the upper (U) and lower (I) level densities as $n_{U}-g_{U} n_{L} / g_{L} ; g_{U}$ and $g_{L}$ are the degeneracies; $b \equiv 1+$ $\mathrm{B}_{U} / \mathrm{g}_{\mathrm{L}} ; \sigma_{\mathrm{RP}}$ is the stimulated emission cross section for the U $\rightarrow \mathrm{L}$ transition. By appropriate scaling of the dependent variables $I$ and $n$, the nonlinear partial differential equations take the form

$$
\frac{\partial y}{\partial z}=x y, \frac{\partial x}{\partial t^{\prime}}=-x y,
$$


which have the general solution first presented by E. 0 . Schulz-DuBois and Beliman, Birnbaum, and Wagner: ${ }^{4,5}$

$$
\begin{aligned}
& \mathbf{x}=-\frac{\partial \Phi}{\partial \mathbf{z}^{\prime}}, \quad \mathbf{y}=\frac{\partial \Phi}{\partial t^{\prime}} \\
& \Phi=\ln \left(F\left(t^{\prime}\right)+G\left(\mathbf{z}^{\prime}\right)\right) .
\end{aligned}
$$

$F$ and $G$ are arbitrary functions that are determined by the initial conditions. The pulse shape is not of particular interest here, so the timeintegrated intensity, or flux,

$$
F=\int_{-\infty}^{\infty} d t I,
$$

which is in untts of energy per area, is used. One can show that the output flux from an amplifier of small signal gain $G^{88}=\exp \left(n \sigma_{s e} I\right)$, is given by

$$
F_{\text {out }}=F_{s} \ln \left[G^{88}\left(\exp \left(F_{\text {In }} / F_{s}\right)-1\right)+1\right] \text {. }
$$

$F_{\text {in }}$ is the incident flux and $F_{s}$ is the saturation flux:

$$
F_{s} \equiv \frac{h \nu}{b \sigma_{s e}}
$$

Various limits and forms of eq. (2) are useful for amplifier analysis. First of all, there are the small signal and large signal limits:

$$
\begin{aligned}
& F_{\text {out }}=\underset{F_{\text {in }} \rightarrow 0}{G^{88} F_{\text {in }}} \\
& F_{\text {out }}=\underset{F_{\text {in }} \rightarrow \infty}{=} F_{\text {In }}+F_{s} \ln G^{88}=F_{\text {in }}+h \nu \frac{n L}{b} .
\end{aligned}
$$


In the second place, one can derive a simple expression for the incident flux required to extract a fraction $\theta$ of the extractable stored energy:

$$
F_{\theta}=F_{s} \ln \left[\frac{G^{8 s}-1}{G^{8 s}-\left(G^{8 s}\right)^{\theta}}\right] \text {. }
$$

If $G^{S S} \gg 1$, which is almost always the case, the flux required to extract one-half the maximum possible amount is given by

$$
F_{1 / 2}=F_{s} / \sqrt{G^{\mathrm{SS}}}
$$

If the laser beam is not diverging strongly, and by this is meant that the beam diameter is not changing by more than a few percent within a given amplifier, the previous eqs. (1) - (4) apply to a given radius within the transverse span of the beam. Thus, all fluxes depend on radius $r$ and the pulse energy is obtained by integration:

$$
E=2 \pi \int_{0}^{\infty} \operatorname{rdr} F(r)
$$

The radial integral over flux cannot be performed analytically, even in the case of an $r$-independent inversion and an incident Gaussian beam. Thus the energy is evaluated numerically before and after each amplifier pass. The fluxes are evaluated on a radial grid consisting of more than 100 points within the radial span of the pulse.

\section{B. Atomic Iodine Transition Parameters}

There are six hyperfine transitions in atomic iodine, each of which:is pressure broadened by Ar at the rate of $\sim 6 \mathrm{MHz} /$ Torr. ${ }^{7,8}$ The oscillator 
typically runs only on the ${ }^{2} P_{1 / 2}(F=3) \rightarrow{ }^{2} P_{3 / 2}(F=4)$ line. The small signal gain cross section is evaluated at the oscillator frequency by considering all six transitions and their appropriate overlap (although small at Ar pressures $\leq 1250$ Torr) with the oscillator frequency. The appropriate upper state populations are likewise included. There are two upper state levels, ${ }^{2} \mathrm{P}_{1 / 2}(\mathrm{~F}=3)$ and ${ }^{2} \mathrm{P}_{1 / 2}(\mathrm{~F}=2)$. Only the former contributes significantly to the energy extraction at the $F=3$ to $F=4$ wavelength at amplifier pressures $\leq 1250$ Torr, so the effective inversion number density is only $\left(\mathrm{g}_{\mathrm{F}}=3\right) \mathrm{n} /\left(\mathrm{g}_{\mathrm{F}}=3^{+} \mathrm{g}_{\mathrm{F}}=2\right)=\frac{7}{12} \mathrm{n}$. Recall that $\mathrm{n}$ is the total inversion number density in the ${ }^{2} P_{1 / 2}$ manifold. The degeneracy of the ${ }^{2} P_{1 / 2}(F=3)$ level is 7 , and this is taken to be $\xi_{U}$. The effect of a two-line oscillator is discussed further in section $V$, but briefly it would be to increase the effective inversion number density to $n$ with degeneracy 12 .

The lower ${ }^{2} \mathrm{P}_{3 / 2}$ hyperfine levels are strongly coupled by collisions with Ar, which also create the line broadening. At a pressure of 600 Torr, the characteristic time for mixing the lower hyperfine levels is $0.04 \mathrm{nsec} .9$ Thus it is assumed that the lower state degeneracy $g_{L}=g\left({ }^{2} P_{3 / 2}\right)=24$, i.e. the entire lower ${ }^{2} \mathrm{P}_{3 / 2}$ manifold is coupled into a single state. These choices for $g_{U}$ and $g_{L}$ and the exact evaluation of $G^{s s}$ allow the inclusion of all the important properties of the six-level system into the two-level analytic solution given in the previous sub-section.

No kinetic pumping or deactivation terms are included in eq. (1). These are not important in the lodine system for the present pumping and extraction time scales.

\section{Beam Propagation}

The oscillator in SAIL-I in its current form emits a beam very similar to a TEM ${ }_{O O}$ Gaussian with a Rayleigh range $\left(\mathrm{z}_{0}=\pi \mathrm{w}_{0}^{2} / \lambda\right)$ of $4.09 \mathrm{~m}$, a waist 
$\left(w_{0}\right)$ of $1.31 \mathrm{~mm}$, and a divergence $\left.\left(\theta_{\mathrm{d}}=\lambda / \pi \mathrm{w}_{0}\right)\right)$ of $320 \mu \mathrm{rad}$. The oscillator beam diameter varies with distance $z$ from the oscillator as 10

$$
w(z)=w_{0}\left(1+\left(z / z_{0}\right)^{2}\right)^{1 / 2}
$$

In addition to any beam expansion produced by a telescope, the natural beam expansion is accounted for by evaluating $w(z)$ at the midpoint of each amplifier (preamplifier) and correcting the radial flux distribution by Lle approprlate expansion factor. For example, referring to Fig. 1 , the diameter at the preamplifier (PAI) is $2.3 \mathrm{~mm}$, and the beam expands $a$. factor of 1.42 upon reaching the beam expander (BE) position. There the beam expander introduces an additional expansion of $7.5 \mathrm{X}$ and is assumed to collimate the beam through the main amplifier. All of the distances used in this study are given in Figs. 1-3 and may be used in conjunction with eq. (5) to evaluate the oscillator beam radius at the varlous elements in the system. All of the amplifiers are assumed to have operating characteristics and dimensions similar to those of the current, system. Dne difference is that the $75 \mathrm{~mm}$ amplifier will have an active length of $3.52 \mathrm{~m}$ instead of the present $3.12 \mathrm{~m}$ when new flashlamps are installed. Table 1 gives the operating characteristics for the preamplifier stages, as well as the variation of small signal gain with Ar pressure. Table 2 presents the same data for the $S A \Pi-175 \mathrm{~mm}$ main amplifier. The Faraday rotators do not enter into the calculations except that they are assumed to have a transmission of 0.75 . 


\section{LAYOUT OF ONE PREAMP SYSTEM}

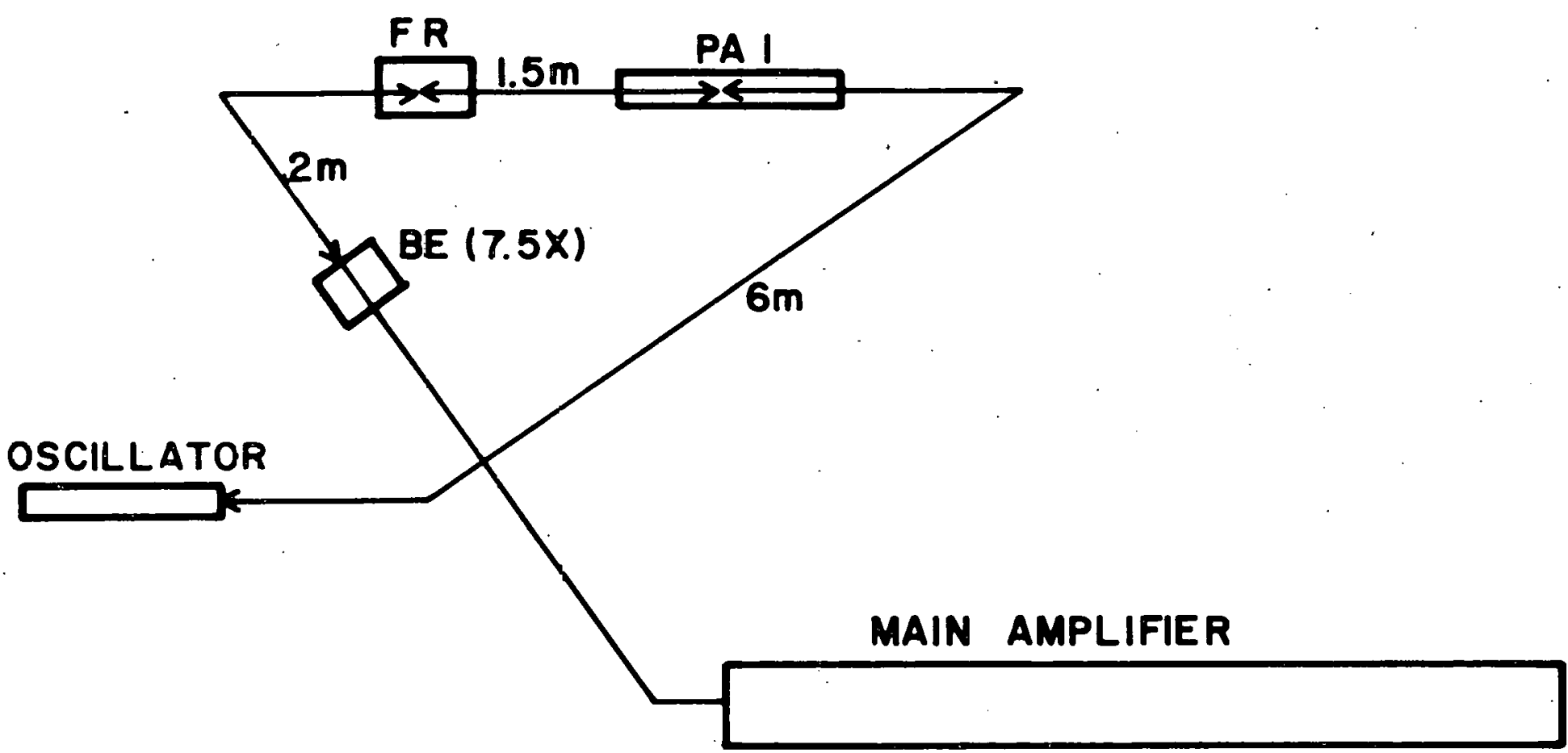

Figure 1. Assumed configuration of system with one preamplifier in chain. PA, FR, and BE denote preamplifier, Faraday rotator and beam expander. Distances between elements are indicated. 


\section{LAYOUT OF TWO PREAMP SYSTEM}

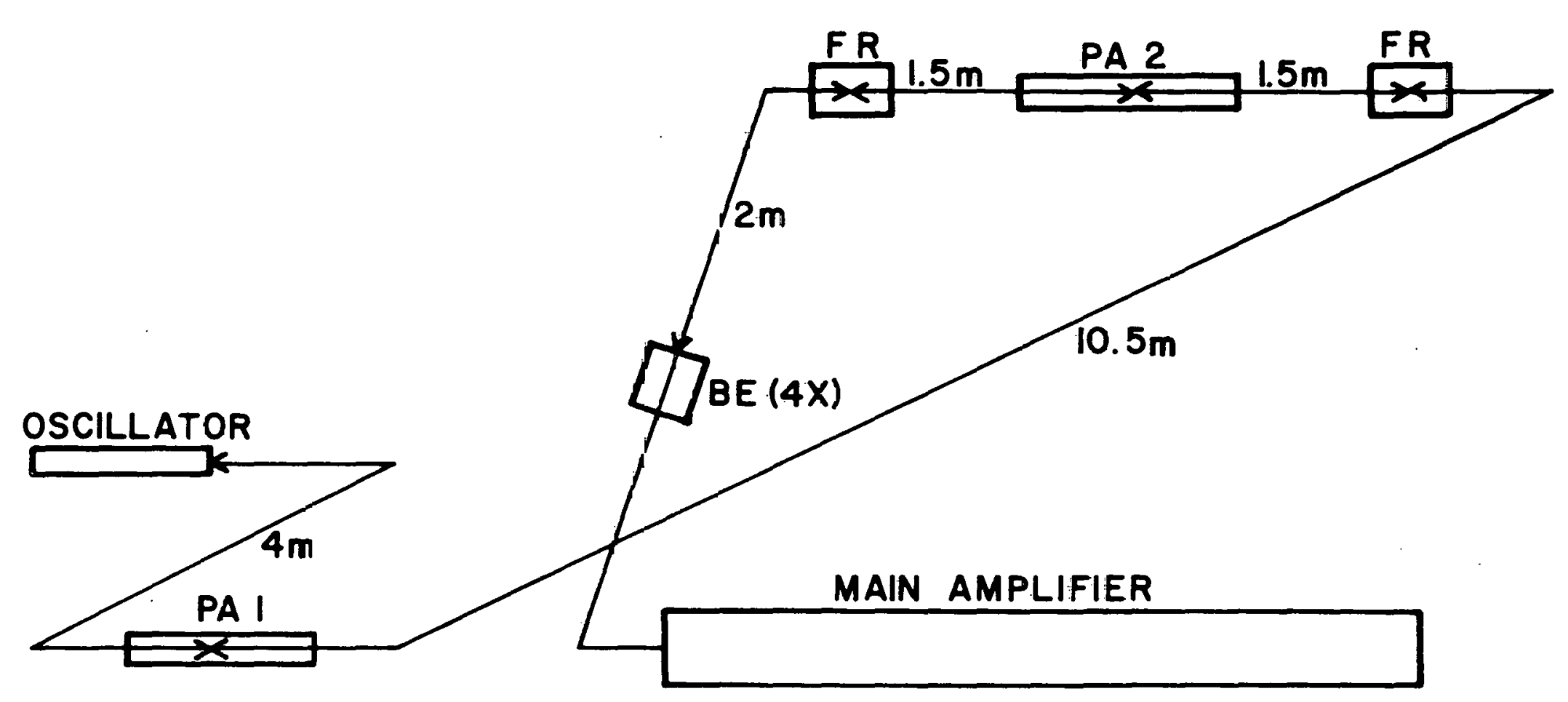

Figure 2. f.ssumed configuration af system with two stages of preamplification. PA, FR, and $B E$ denote preamplifier, Faraday rotator, and beam expander. 


\section{LAYOUT OF THREE PREAMP SYSTEM}

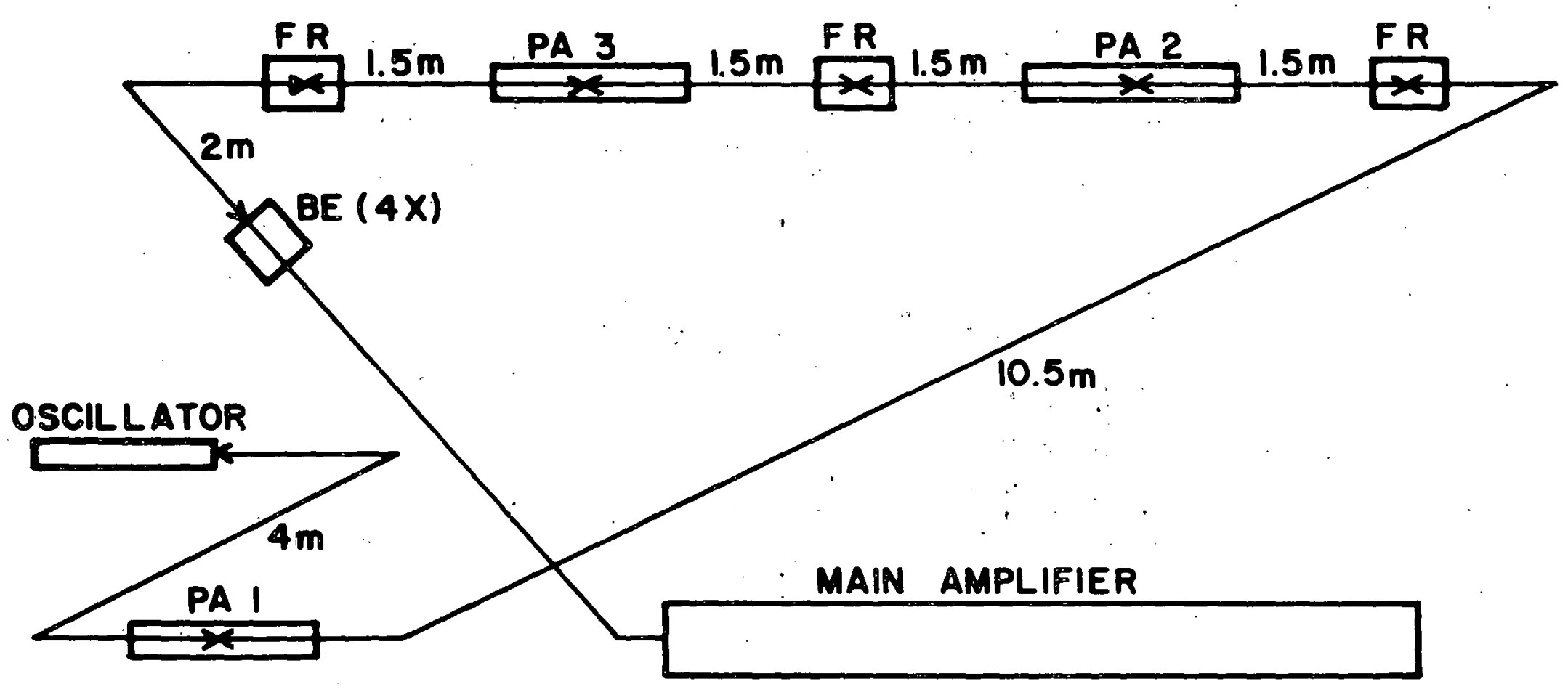

Figure 3. Assumed configuration of system with three stages of preamplification. $\mathrm{PA}, \mathrm{FR}$, and $\mathrm{BE}$ denote preamplifier, Faraday rotator, and beam expander. 


\begin{tabular}{|c|c|c|c|}
\hline Length, act & & $.88 n$ & \\
\hline Radius, act & & .008 & \\
\hline Inversion, & & 2.45 & $0^{17} / \mathrm{cm}^{3}$ \\
\hline Extractable & ergy & 4.365 & \\
\hline Extractable & $3-4$ & 2.957 & \\
\hline $\mathrm{P}_{\mathrm{Ar}}$ (Torr) & $G^{s s}$ & $F_{s}\left(J / \mathrm{cm}^{2}\right)$ & $F_{1 / 2}\left(\mathrm{~J} / \mathrm{cm}^{2}\right)$ \\
\hline 750 & 1275 & .21 & .006 \\
\hline 1000 & 310 & .26 & .015 \\
\hline 1250 & 134 & .30 & .026 \\
\hline
\end{tabular}

Table 1. Specifications of the preamplifier stages used in the calculation. The small signal gain $G^{s s}$, the saturation flux $F_{S}$, and the flux to half saturate $F_{1 / 2}$, are all ealculated for an oscillator operating on the $F=3$ to $F=4$ line. 


$$
\begin{aligned}
& \text { Length, active } \\
& \text { Radius, active } \\
& \text { Inversion, total } \\
& \text { Extractable energy } \\
& \text { Extractable on } 3-4
\end{aligned}
$$

$3.52 \mathrm{~m}$

\begin{tabular}{|c|c|c|c|}
\hline $\mathrm{P}_{\mathrm{Ar}}$ (Torr) & $G^{s . s}$ & $F_{s}\left(\mathrm{~J} / \mathrm{cm}^{2}\right)$ & $\mathrm{F}_{1 / 2}\left(\mathrm{~J} / \mathrm{cm}^{2}\right)$ \\
\hline 600 & 1113 & .17 & .005 \\
\hline 800 & 256 & .22 & .014 \\
\hline 1000 & 108 & .26 & .025 \\
\hline
\end{tabular}

$.03 \mathrm{~m}$

$0.5 \times 10^{17} / \mathrm{cm}^{3}$

$50.11 \mathrm{~J}$

$33.94 \mathrm{~J}$

Table 2. Specifications of $75 \mathrm{~mm}$ ID main amplifier used in the calculation. The small signal gain $G^{8 s}$, the saturation flux $F_{s}$, and the flux to half saturate $F_{I / 2}$ are all calculated for an oscillator operating on the $F=3$ to $F=4$ line. 
III. Parametric Study of Energy Extraction in Upgraded SAIL-I

In order to assess the potential of the SAIL-I facility, the following survey of operating parameters has been performed. First, the oscillator output energy was varied from 1 to 3 to $5 \mathrm{~mJ}$, with a beam as described in the previous section. Second, either one, two, or three preamplifiers were included in the optical chaln. Thlrd, the Ar pressure in the preamplifiers was varied over 750,1000 , and 1250 Torr, with the associated variation in the small signal, gain as given in Tablo 1 . Fourth, the Ar pressure in the main amplifier was varied over 600,800 , and 1000 Torr, with the associated small signal gain variation as given in Table 2 . The magnification of the single beam-expander element in each layout (Figs. 13) was adjusted for rough maximization of the final output energy. The beams are strongly apertured at a f'ew places, and always at the entrance to the main amplifier. Soft apertures will be necessary to retain good beam quality.

Tables 3-5 present the output energies expected from one, two, and three preamplifier systems, respectively, for various preamplifier and $75 \mathrm{~mm}$ amplifier pressures. The oscillator output energy affects the total output very little, except in the low gain (high pressure), single preamplifier case. This is because of the large amount of saturation in the system. In fact, in the three preamplifier system, the oscillator energy had almost no effect on the final output. Figure 4 summarizes most of this information and also plots the output energy vs input energy to the main amplifier alone. It is judged that two preamplifiers are required for moderately efficient extraction, and that a third preamplifier would add only $\sim 5 \mathrm{~J}$ to the output. 
Alternative schemes and upgrading modifications to achieve higher output energy are discussed in section $\mathrm{V}$. 


\begin{tabular}{|c|c|c|c|}
\hline $\begin{array}{l}\text { Preamplifier } \\
\text { Pressure } \\
\text { Main Amplifier } \\
\text { Pressure }\end{array}$ & 750 & 1000 & 1250 \\
\hline $\sin$ & $\begin{array}{l}17.9 \\
17.3 \\
15.8\end{array}$ & $\begin{array}{l}16.8 \\
15.9 \\
13.5\end{array}$ & $\begin{array}{l}15.7 \\
1 ! 1.5 \\
11.6\end{array}$ \\
\hline 800 & $\begin{array}{l}12.9 \\
12.2 \\
10.5\end{array}$ & $\begin{array}{r}11.6 \\
10.7 \\
8.3\end{array}$ & $\begin{array}{r}10.5 \\
9.3 \\
6.5\end{array}$ \\
\hline 1000 & $\begin{array}{l}9.2 \\
8.6 \\
7.0\end{array}$ & $\begin{array}{l}8.1 \\
7.2 \\
5.2\end{array}$ & $\begin{array}{l}7.0 \\
6.0 \\
3.8\end{array}$ \\
\hline
\end{tabular}

Table 3. Calculated energy output ( $J$ ) for the one preamplifier system. The different energles in each box correspond to an oscillator output of 5,3 , and $1 \mathrm{~mJ}$. All energies reflect single line $F=3$ to $F=4$ operation of system. 


\begin{tabular}{|c|c|c|c|}
\hline $\begin{array}{l}\text { Preamplifier } \\
\text { Pressure } \\
\text { Main Amplifier } \\
\text { Pressure }\end{array}$ & 750 & 1000 & 1250 \\
\hline 600 & $\begin{array}{l}27.5 \\
27.4 \\
27.1\end{array}$ & $\begin{array}{l}26.9 \\
26.7 \\
26.1\end{array}$ & $\begin{array}{l}26.2 \\
25.8 \\
24.7\end{array}$ \\
\hline 800 & $\begin{array}{l}24.6 \\
24.4 \\
24.0\end{array}$ & $\begin{array}{l}23.8 \\
23.5 \\
22.8\end{array}$ & $\begin{array}{l}22.9 \\
22.5 \\
21.1\end{array}$ \\
\hline 1000 & $\begin{array}{l}21.9 \\
21.7 \\
21.3\end{array}$ & $\begin{array}{l}21.0 \\
20.7 \\
19.9\end{array}$ & $\begin{array}{l}20.0 \\
19.5 \\
18.0\end{array}$ \\
\hline
\end{tabular}

Table 4. Calculated energy output (J) for the two preamplifier system. The different energies in each box correspond to an oscillator output of 5,3 , and $1 \mathrm{~mJ}$. All energies reflect single line $F=3$ to $F=4$ operation of system. 


\begin{tabular}{|c|c|c|l|}
\hline $\begin{array}{c}\text { Preamplifier } \\
\text { Pressure } \\
\begin{array}{c}\text { Main Amplifier } \\
\text { Pressure }\end{array}\end{array}$ & 750 & 1000 & 1250 \\
\hline 600 & 31.5 & 31.1 & 30.6 \\
\hline 800 & 28.9 & 28.5 & 27.9 \\
\hline 1000 & 26.5 & 26.0 & 25.4 \\
\hline
\end{tabular}

Table 5. Calculated energy output ( $J$ ) for the three preamplifier system. The oscillator output is $1 \mathrm{~mJ}$. All energies reflect single line $F=3$ to $F=4$ operation of the system. 
Figure 4. Performance curves of the main amplifier for three different Ar pressures. The ranges of input energy to the main amplifier that may be achieved with one, two, or three preamplifiers (PA) are indicated. Ar pressure in the preamplifier chain is denoted by: - 1250 Torr, $\mathbf{\Delta}-1000$ Torr, $\mathbf{\square}-750$ Torr. The oscillator output energy is $1 \mathrm{~mJ}$ and single line $\mathrm{F}=3$ to $F=4$ operation is assumed.

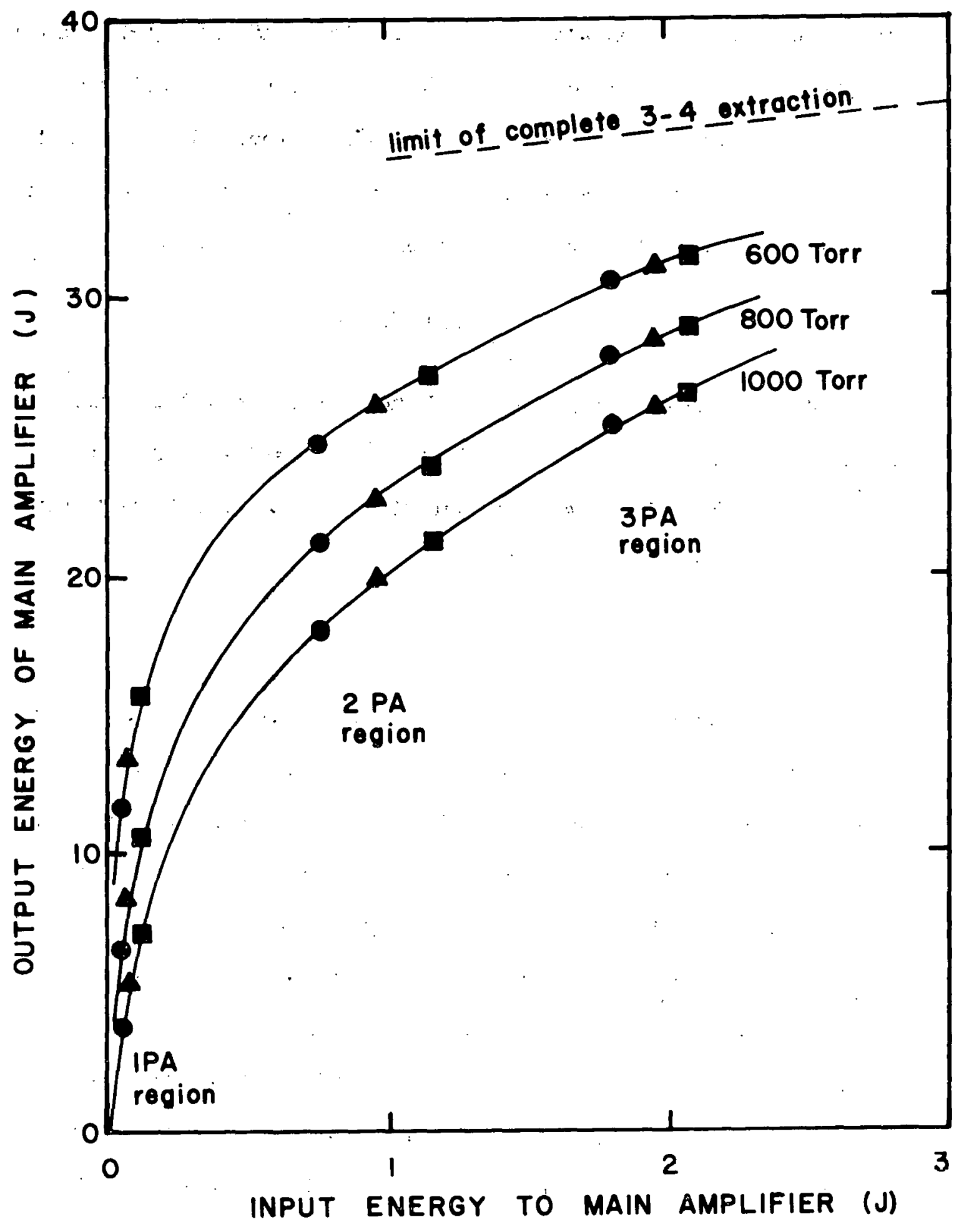


IV. Beam Quality Limitations

\section{A. Prelase and Self-Oscillation}

The Plasterix iodine laser ${ }^{11}$ at Max-Planck-Instltut für Plasmaphysik is an iodine laser system with two preamplifiers and one final amplifier. The output is 30 joules in $600-800$ psec. Prelase, as distinguished from self-oscillation, is unimportant in this system. To estimate the amount of prelase in the SAIS-1 system, consider the power radiated into a solid angle $A \cap$ by a volume $V$ of emitters of number density $n$ :

$$
P=n V A_{s p} \Delta \Omega h \nu /(4 \pi)
$$

$\mathrm{A}_{\mathrm{sp}}$ is the spontaneous decay rate. If the dimensions and inversion characteristics of a preamplifier (Table l) are used and if $\Delta \Omega$ is assumed to be close to the solid angle characteristic of the oscililator beam divergence, one obtains

$$
\mathrm{F} \equiv 10^{-7} \mathrm{~W}
$$

If the total small signal gain is $G^{\text {tot }}$ and the delay time from pumping to pulse extraction is on the order of $10 \mu \mathrm{sec}$ as is currently the case, an energy of $<10^{-12} \mathrm{G}$ tot $\mathrm{J}$ will be delivered to the target prior to the main pulse. Thus, the four-stage system (three preamplifiers and main amplifinr) with a small elgnal gain of $100 /$ stage would dellver $<10^{-4} \mathrm{~J}$ prelase; if the gain is 1000/stage, there will be on the order of a joule of prelase. Target isolators would be necessary in the latter case.

The likelihood of self-oscillation is difficult to predict in the system. The principal allowance that has been made for it is to include a range of small signal gain for each amplifier stage in the survey reported in section 
III. A value of $G^{s 8}$ as low as 100/stage shoula not produce self-oscillation in the amplifiers. A value of $G^{8 S}$ as large as 1000/stage, however, will probably result in some self-oscillation of the amplifiers. The experience obtained by the German group on Plasterix indicates that the small signal gains may have to be kept as low as 100/stage to suppress selfoscillation. The SAIL-I system has been run with a small signal gain of 100 in the preamplifier and 2700 in the $75 \mathrm{~mm}$ amplifier with no isolators; the amplifiers did not self-oscillate in this configuration. However, when the gain was increased slightly in elther the preamplifier or the main amplifier, self-oscillation occurred. At these gains, prelase due to amplified spontaneous emission wäs negliglble. Hence, both in Plasterix and in SAII-1, self-oscillation of the amplifiers is the limiting factor. That is why Faraday rotators are included in the upgrading study in section III.

\section{B. Target Feedback}

Back-reflection from the target is not so severe a problem for the lodine laser system as for glass laser systems, because the medium is not subject to damage. Windows and other optical elements are, however, so the problem cannot be ignored. It is anticipated that the large amount of saturation present in the main amplifier will reduce the backward pass gain to a sufficiently low value that damage will not occur. From FIg. 4 one finds that the gain is on the order of 20 for two-preamplifier operation. This is low enough that the back-reflected radiation should not damage the entry windows of the main amplifier. The remaining part of the system is protected by the Faraday rotator after the final preamplifier: If the target is highly reflective in its initial state (i.e. a metallic target), 
a Faraday rotator may be necessary after the $75 \mathrm{~mm}$ amplifier to prevent the main amplifier from self-oscillating. For the purpose of this report, it is assumed that a final Faraday rotator will not be necessary. Ensuing developments may change this viewpoint.

\section{Aperturing}

The beam will be hard-apertured at several points in the system. This would introduce distortions in the phasefront due to diffraction. While it is true that catastrophic self-focussing due to nonlinear effects would most likely not be a problem, the focussability of the beam at the target wouid definitely be affected.

One method to eliminate the hard aperturing is to use apodized or "soft" apertures. The soft apertures produced at Sandia Laboratories" 12 would probably not be capable of withstanding the high power and energy densities of a large iodine laser, especially in the later stages. Soft apertures capable of withstanding high power and energy densities can probably be supplied by commercial vendors. ${ }^{13}$ of course, soft, apertures are an additional source of loss in the system, and if the requirement for extremely tight focussing necessitates the use of soft apertures, the expected output energies tabulated in section III might be lowered slightly. However, the calculations have used an effective radius smaller than the inner alameter of the ampliflers in all cuses. 
V. Additional Possibilities for Increasing the Output Energy From SAII-I

A. Two-Line Oscillator

In the preceding calculations it was assumed that the oscillator output was purely the $F=3$ to $F=4$ hyperfine transition. This input will extract energy from only the $F=3$ upper level. At the pressures considered in this report, there is little overlap of the three hyperfine transitions starting from the $F=2$ upper level with the $F=3$ to $F=4$ transition. However, $5 / 12$ of the inversion density is stored in the $F=2$ upper level. Extracting this energy would increase the extraction efficiency by $30-50 \%$.

One means of extracting this energy is to make the oscillatior output. spectrum have a component at the $F=2$ to $F=2$ transition wavelength. This could be accomplished by running two oscillators simultaneously, one at the $F=3$ to $F=4$ transition and the other at the $F=2$ to $F=2$ transition. The wavelength of each oscillator can be controlled with a Fabry-Perot etalon placed in the cavity. ${ }^{14}$ The $F=3$ to $F=4$ oscillator could be run slightly off line center, so that the two components have equal gain in the amplifiers. The gain would still be comparable to the range of gains studied in this report.

The main difficulty in using two separate oscillatcrs is to reduce the relative time jitter between the two. The jitter in the present oscillator in SAIL-I has been measured to be $\pm 30 \mathrm{nsec}$. Part of this is jitter in the breakdown of the spark gap and of the flashlamps, and part is in the statistical nature of the build-up of the laser radiation from noise. The first source of jitter can be eliminated by pumping both oscillators by the same flashlamps, i.e. by placing both laser tubes in the same head. The second source of jitter can be eliminated by chopping out the $1-10$ nsec output pulse from both oscillators with the same optical 
gate. Pulses with 50 nsec duration can be produced routinely by gainswitching the oscillators with high cavity loss (this can be accomplished by the mode-selecting aperture alone). Hence the degree of pulse cverlap should be high, and the optical gate can select 1-10 nsec simultaneous slices from both oscillator autputs.

One question that remains to be answered by experiment is the relative amplitude stability of the two oscillators. The amplitude stability of the present oscillator is better than $\pm 10 \%$, but slight thermally-induced shifts in the etalon frequencies may degrade the amplitude stability somewhat. Since the final amplifier will be heavily saturated, the final out put energy should be relatively insensitive to the oscillator output energy, as is shown in tables $3-5$.

\section{B. Improved Pumping and Energy Storage in $75 \mathrm{~mm}$ Amplifier}

The survey study reported in Section III assumed that the pumping of the main amplifier produced an inversion density of $0.5 \times 10^{17} / \mathrm{cm}^{3}$, the current experimentally confirmed value. This corresponds to an extractable energy density of about $5 \mathrm{~J} / \mathrm{l}$ or $3.4 \mathrm{~J} / \mathrm{l}$ on the $3-4$ transition. The inversion density of the main amplifier was originally $0.7 \times 10^{17} / \mathrm{cm}^{3}$. The decrease in inversion density is probably due to degradation of the f'lashlamps or flashlamp reflectors, or to contamination of the mix tank due to reaction of $\mathrm{C}_{3} \mathrm{~F}_{7} \mathrm{I}$ with the nickel-plated walls. The latter has been confirmed for the case of stainless steel mix tank walls.

The energy storage may, in fact, be doubled if the $\mathrm{C}_{3} \mathrm{~F}_{7} \mathrm{I}$ pressure is increased. However, this produces a very strong gain-vs-radius profile and would require a careful study of radial beam tailoring to allow for large-radius, high-intensity radial intensity gradients and, strong aper- 
turing near the amplifier outer radius. In practice, it is doubtful that more than a $50 \%$ overall increase in main amplifier energy storage can be obtained at this time.

\section{Cassegrainian Optics}

Cassegrainian optics or other schemes to multiple-pass the $75 \mathrm{~mm}$ amplifier can, in principle, increase the energy extraction of the amplifier. Unfortunately, they suffer from two disadvantages. First, efficient overlap of the multiple passes is difficult. If the beams are comparable in size to the amplifier bore, the reflecting elements severely aperture the beam. Second, and most serious, due to its high aspect ratio, placing elements with high feedback at the output end of the $75 \mathrm{~mm}$ amplifier cause it to self-oscillate at quite low gains. In the case that only the target plasma is highly reflective, the feedback occurs only after the main pulse has saturated the amplifier, thus lowering the gain. It is not expected that multiple-pass schemes (for which the feedback is always high) will be a practical method for efficient energy extraction from the $75 \mathrm{~mm}$ amplifier. 
In Sandia Internal Report SLA-73-0976, ${ }^{15}$ an error was made in eq. $6 a$. Because of the rapid equilibration of the lower state manifold $n_{\ell}$, the change in the population of upper level $\mathrm{n}_{2}$ is equal to the change in $\mathrm{n}_{\ell}$, not in the individual level $n_{1}$. Hence eq. 6a should read

$$
\begin{aligned}
\frac{d n_{\ell}}{d t} & =w_{21}\left(n_{2}-\frac{g_{2}}{g_{1}} n_{l_{1}}\right) \\
& =w_{21}\left(n_{2}-\frac{g_{2}}{g_{l}} n_{l}\right)
\end{aligned}
$$

Adding this equation to eq. $6 \mathrm{~b}$ yields

$$
\frac{d}{d t}\left(n_{2}-\frac{g_{2}}{g_{l}} n_{l}\right)=-w_{2 l}\left(1+\frac{g_{2}}{g_{l}}\right)\left(n_{2}-\frac{g_{2}}{g_{l}} n_{l}\right)
$$

Following through the remaining equations yields a b-factor of

$$
\mathrm{b}=I+\frac{\mathrm{g}_{2}}{\mathrm{~g}_{\ell}}
$$

not $b=1+\frac{g_{2}}{g_{1}}$ as given in the report. The inversion is still given by

$$
\begin{aligned}
\Delta \mathrm{N} & =\mathrm{n}_{2}-\frac{\mathrm{g}_{2}}{\mathrm{~g}_{\overline{1}}} \mathrm{n}_{1} \\
& =\mathrm{n}_{2}-\frac{\mathrm{g}_{\frac{1}{2}}}{\mathrm{~g}_{\ell}} \mathrm{n}_{\ell}
\end{aligned}
$$

Hence, the effect of the rapid equilibration of the lower state manifold is that the effective lower laser level is the entire manifold $\mathrm{n}_{\ell}$ with degeneracy $g_{l}$, instead of the real lower laser level $n_{1}$ with degeneracy $g_{1}$. The $b$-factor and inversion given above in eqs. $3 a$ and $5 a$ are the ones to be used in eqs. 1, 2, and 3 in Section II. 


\section{REFERENCES}

1. K. J. Witte, G. Brederlow, E. Fill, K. Hohla, and R. Volk, presented at 4 th Int. Workshop on Laser Interaction and Related Phenomena, RPI, Troy, NY, Nov., 1976.

2. V. A. Katulin, V. Yu. Nosach, and A. L. Petrov, Sov. J. Quantum Electron. 6, 998 (1976).

3. J. N. Olsen, J. Appl. Phys. 47, 5360 (1976).

4. E. O. Schulz-DuBois, Bell System Technical Journal 43, 625 (1964).

5. R. Bellman, G. Birnbaum, and W. G. Wagner, J. Appl. Phys. 34,780 (1963).

6. V. S. Zuev, V. A. Katulin, V. Yu. Nosach, and O. Yu. Nosach, Sov: Phys. JETP 35, 870 (1972).

7. W. Fuss and K. Hohla, "Pressure Broadening of the $1.3 \mu \mathrm{m}$ Iodine Laser Line," Max-Planck-Institut für Plasmaphysik Report IPP IV/67, 1974.

8. T. D. Padrick and R. E. Palmer, J. Chem.Phys. 62, 3350 (1975).

9. E. A. Yukov, Sov. J. Quantum. Electron. 3, 117 (1973).

10. A. Yariv, "Quantum Electronics," 2nd ed. (John Wiley \& Sons, New York, 1975), p. 111 .

11. K. Eidmann, Ch. Dorn, and R. Sigel, "Initial Target Experiments with the Iodine Laser," presented at the 10th European Conference on Laser Interaction with Matter, Palaiseau, Oct., 1976; Max-Planck-Institut für Plasmaphysik Report IPP IV/95, 1976.

12. J. P. Anthes, J. W. Lavasek, and M. A. Palmer, "A Picosecond and Nanosecond Nd:Glass High Power Laser Amplifier System," SAND-76-0432, 1976. 
13. CVI Laser Corporation, Albuquerque, N. M., advertises high power apodized apertures.

14. E. S. Mukhtar, H, J. Baker, and T. A. King, Opt. Commun. 19, 193 (1976).

15. R. E. Palmer, "Degeneracy Factọ in Laser Transitions with Nondegenerate Hyperfine Splitting," SLA-73-0976, 1973. 
UNLIMITED RELEASE

DISTR IBUTION:

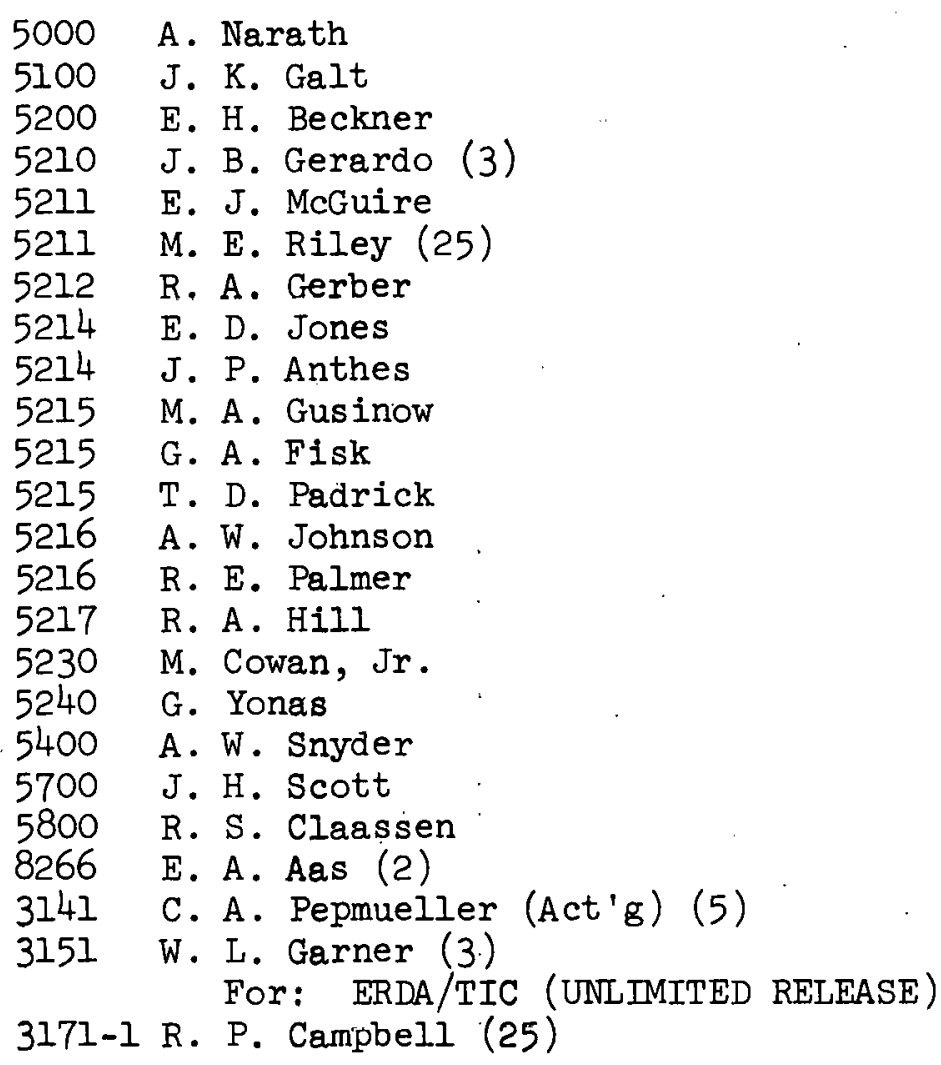

